\title{
Reactive Multilayers and Coatings Fabricated by Spray Assembly: Influence of Polymer Structure and Process Parameters on Multi-Scale Structure and Interfacial Properties
}

Harshit Agarwal, ${ }^{1}$ William M. Breining, ${ }^{2}$ Gabriel Sánchez-Velázquez, ${ }^{1}$ and David M. Lynn ${ }^{1,2, *}$

${ }^{1}$ Department of Chemical \& Biological Engineering, University of Wisconsin-Madison, 1415 Engineering Dr., Madison, WI 53706, USA, ${ }^{2}$ Department of Chemistry, University of WisconsinMadison, 1101 University Ave., Madison, WI 53706, USA. E-Mail: (D.M.L) dlynn@engr.wisc.edu

\section{Supporting Information}

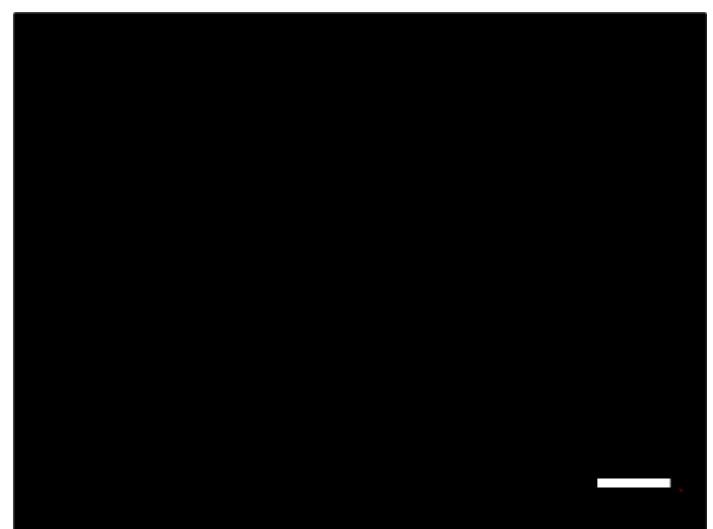

Figure S1. Fluorescence microscopy image of a propylamine-treated P1/PEI film $(\sim 80 \mathrm{~nm}$ thick and fabricated on a silicon substrate) after reactive microcontact printing using a PDMS stamp inked with TMR-cadaverine. No significant fluorescence was observed in this control experiment (see main text) Scale bar $=100 \mu \mathrm{m}$.
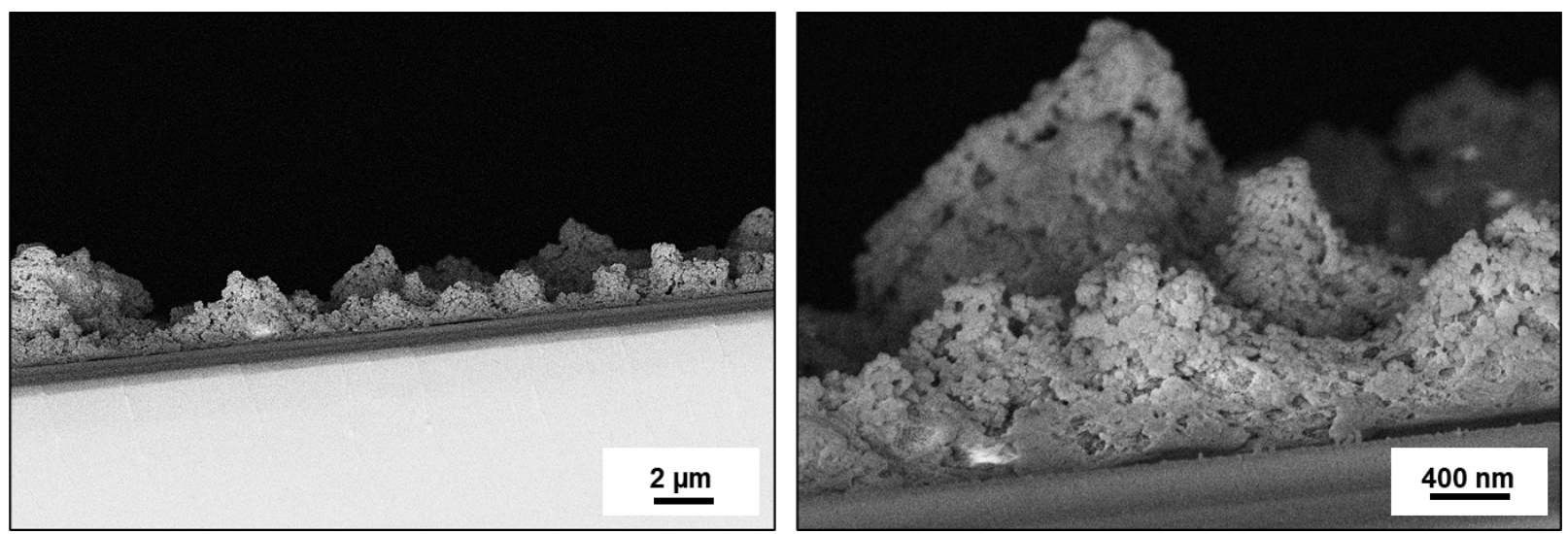

Figure S2. Cross-sectional SEM images of 35 bilayer P1/PEI films fabricated on a silicon substrate by spray-based assembly in the absence of intermittent spray-rinse steps. The substrate was scratched with a diamond-tipped pen on the back and then fractured along the scratch to expose the cross-section prior to imaging. 


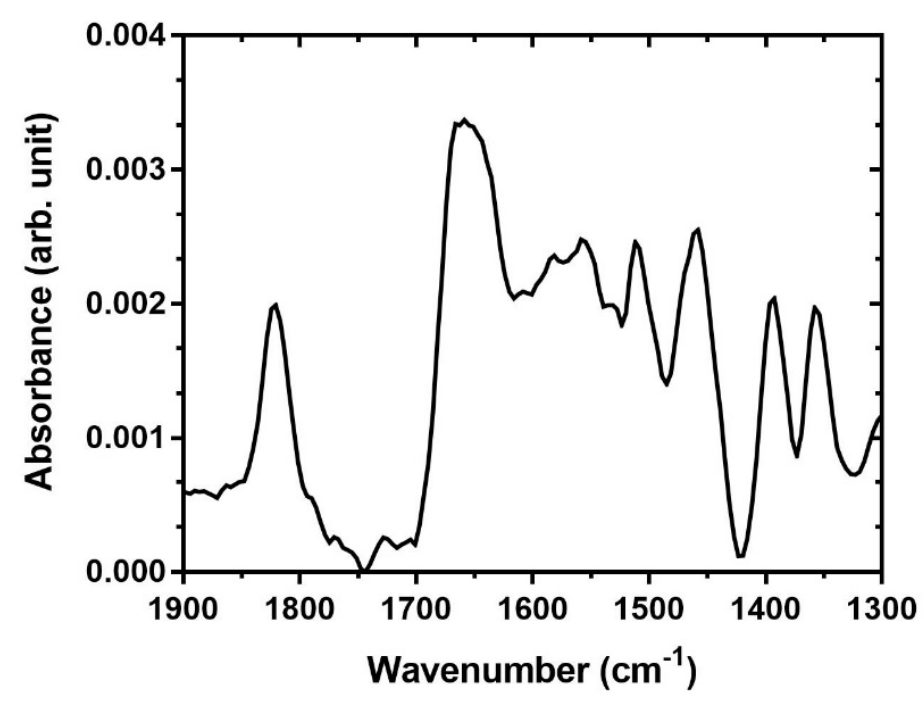

Figure S3. Representative ATR-IR spectrum of a P1/PEI film that was removed from its underlying silicon substrate by scraping.
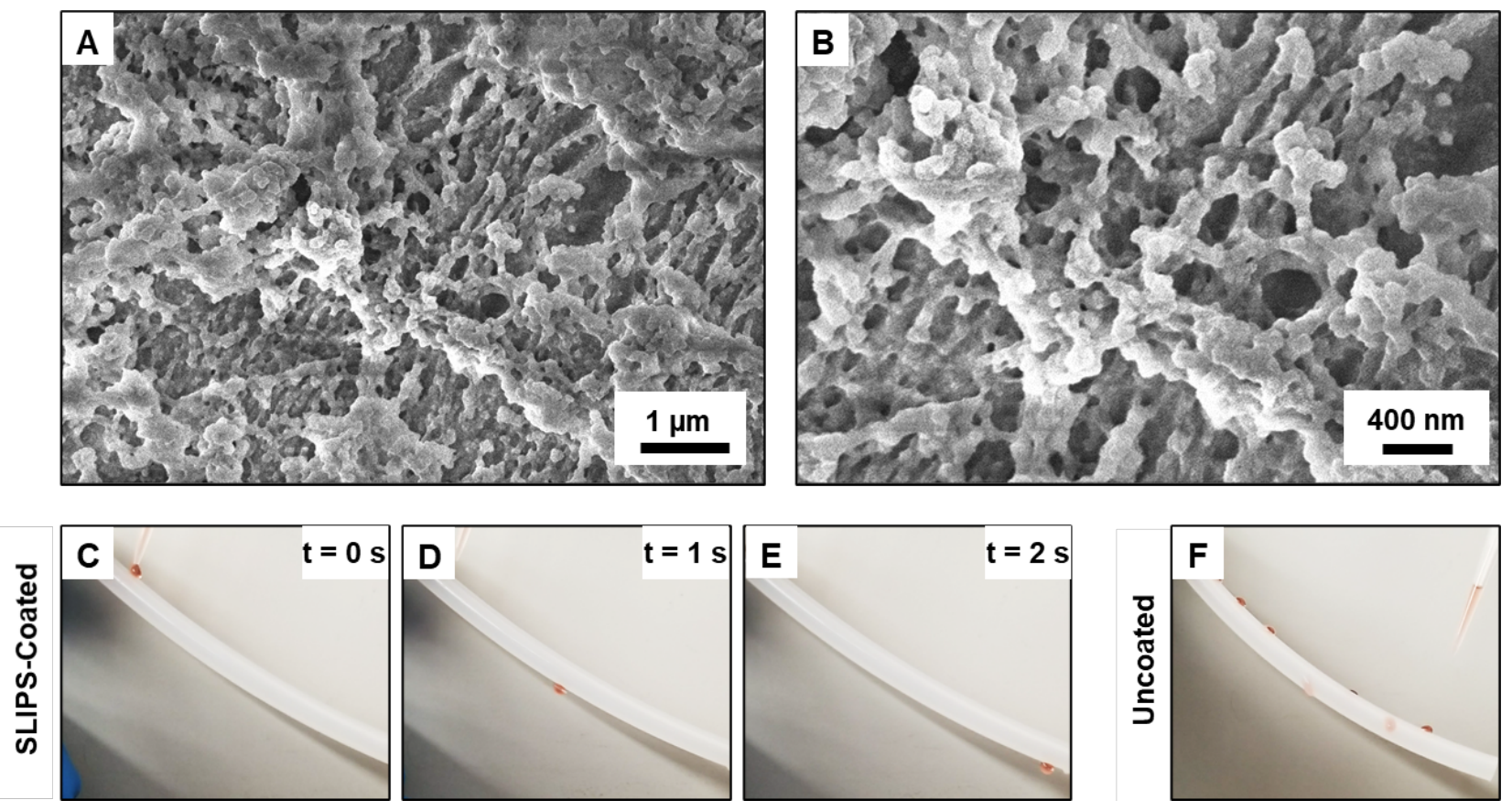

Figure S4. (A,B) Top-down low-magnification (A) and high-magnification (B) SEM images showing micro- and nanoscale roughness and porosity of multilayers (25 bilayers) sprayed onto the outside surfaces of PE tubing segments. (C-E) Series of images showing colored water droplets sliding on the outside surface of SLIPS-coated PE tubing segments. (F) Image showing water droplets stuck on the outside surface of a bare, uncoated PE tubing segment. 
A

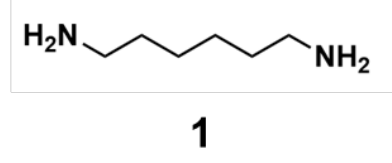

C

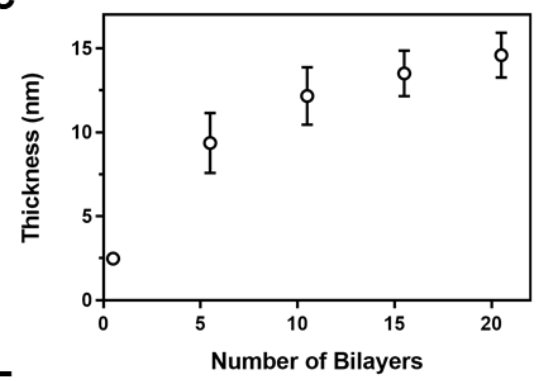

E

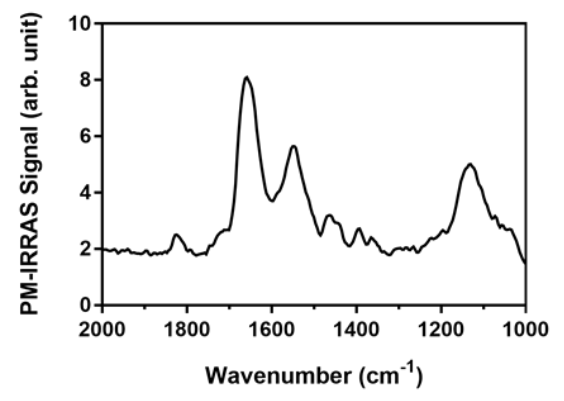

B

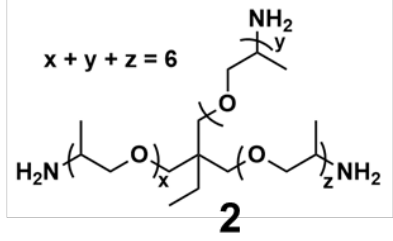

D

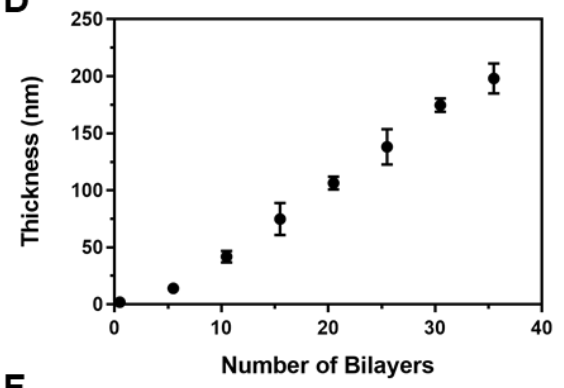

$\mathbf{F}$

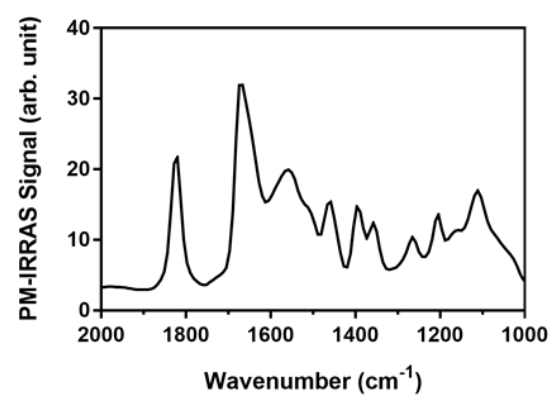

Figure S5. (A,B) Chemical structures of the primary amine-containing small non-polymeric linkers [linker 1 (hexamethylene diamine; difunctional) and linker 2 (Jeffamine ${ }^{\circledR}$ T-403, trifunctional)] used for fabrication of thin reactive films. (C,D) Plot of optical thickness (as determined by ellipsometry) versus the number bilayers of (C) P1/linker 1 and (D) P1/linker 2 sprayed onto planar silicon substrates. (E,F) Plots showing representative PM-IRRAS spectra for (E) P1/linker 1 and (F) P1/linker 2 fabricated on gold-coated silicon substrates. The absorbance peak at $\sim 1826 \mathrm{~cm}^{-1}$ in each spectrum corresponds to the carbonyl group of unreacted azlactone groups present in the films. 

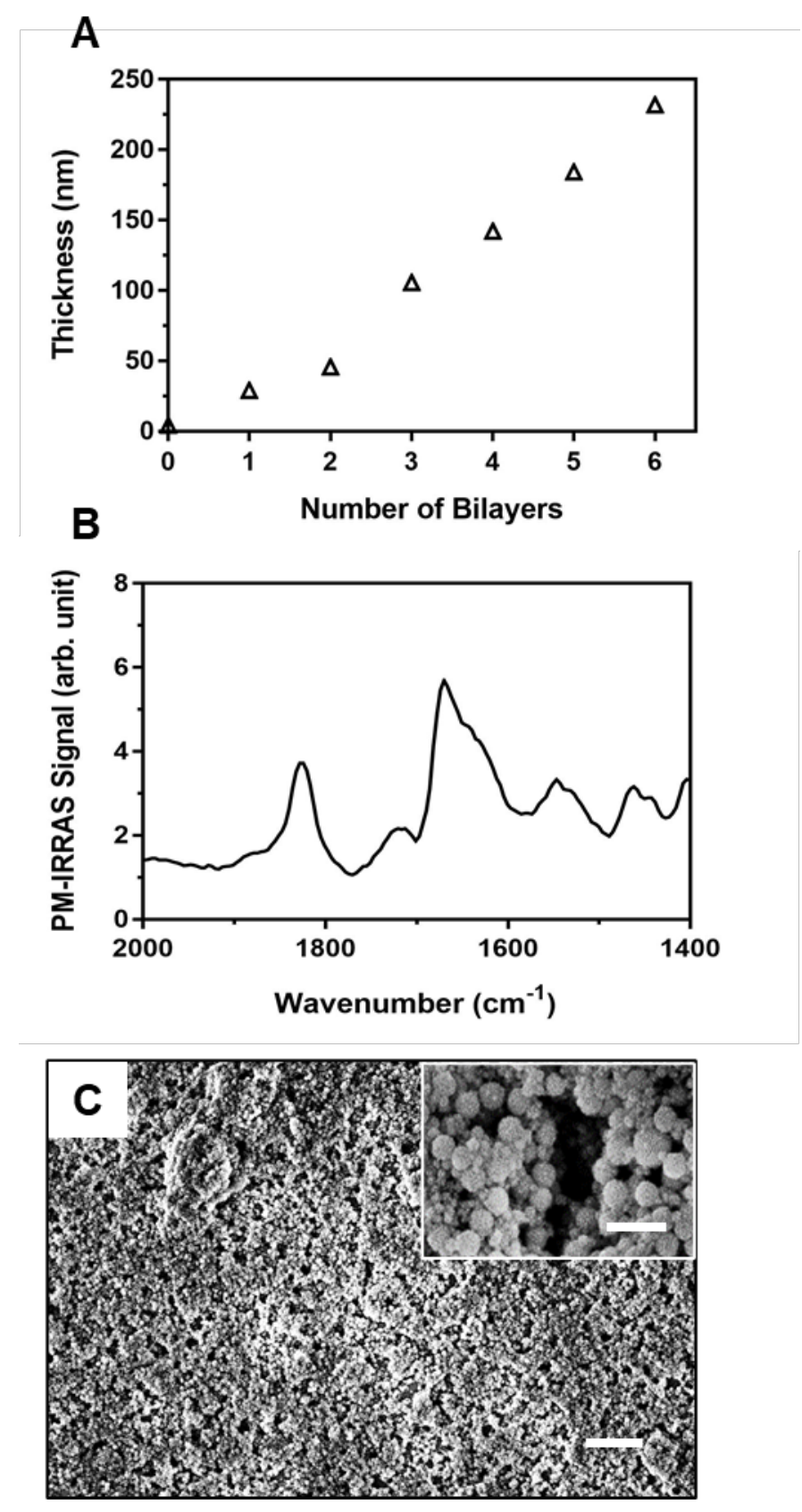

Figure S6. (A) Plot of optical thickness (as determined by ellipsometry) versus the number of bilayers fabricated by sequentially spraying P1 (10mM in DMSO) and amine-functionalized silica nanoparticles ( $1 \mathrm{wt} \%$ in DMSO) on a planar silicon substrate. (B) Plot showing representative PM-IRRAS spectra for P1/silica nanoparticle films fabricated on a gold-coated silicon substrate. The absorbance at $\sim 1826 \mathrm{~cm}^{-1}$ corresponds to the carbonyl group of unreacted azlactone functionality. (C) Representative SEM image of a P1/nanoparticle film showing micro- and nano-scale porosity. Scale bar $=1 \mu \mathrm{m} ; 100 \mathrm{~nm}$ inset. 

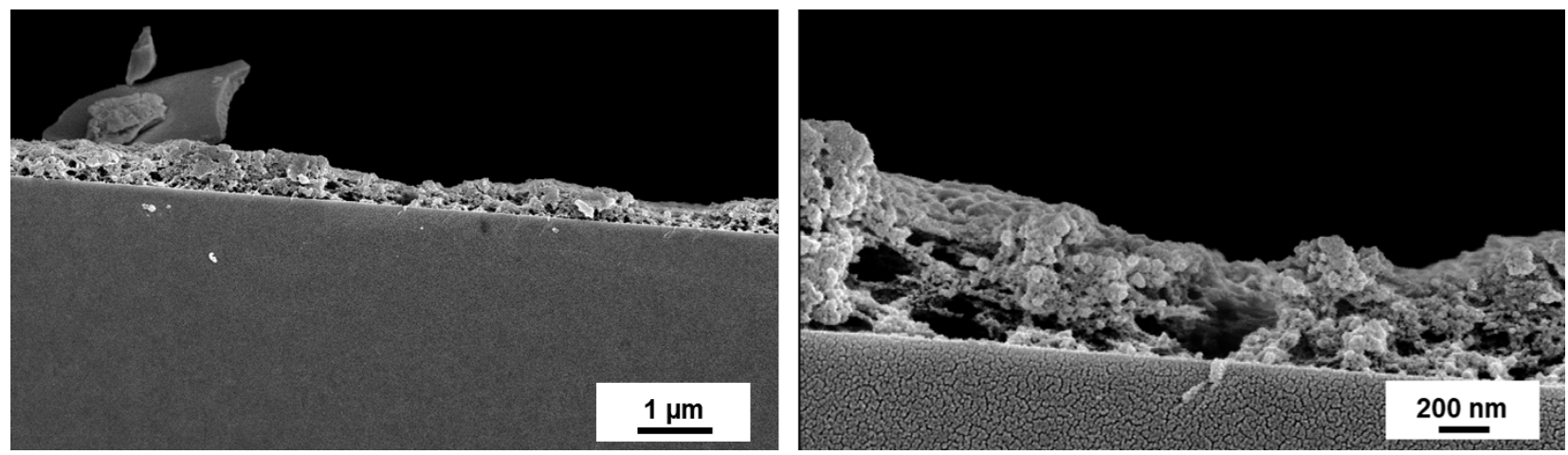

Figure S7. Cross-sectional SEM images of P1/PEI films fabricated on a silicon substrate by simultaneously spraying P1 and PEI (at spraying rate ratio of 1:1) for 145 seconds. The substrate was scratched with a diamond-tipped pen on the back and then fractured along the scratch to expose the crosssection prior to imaging.
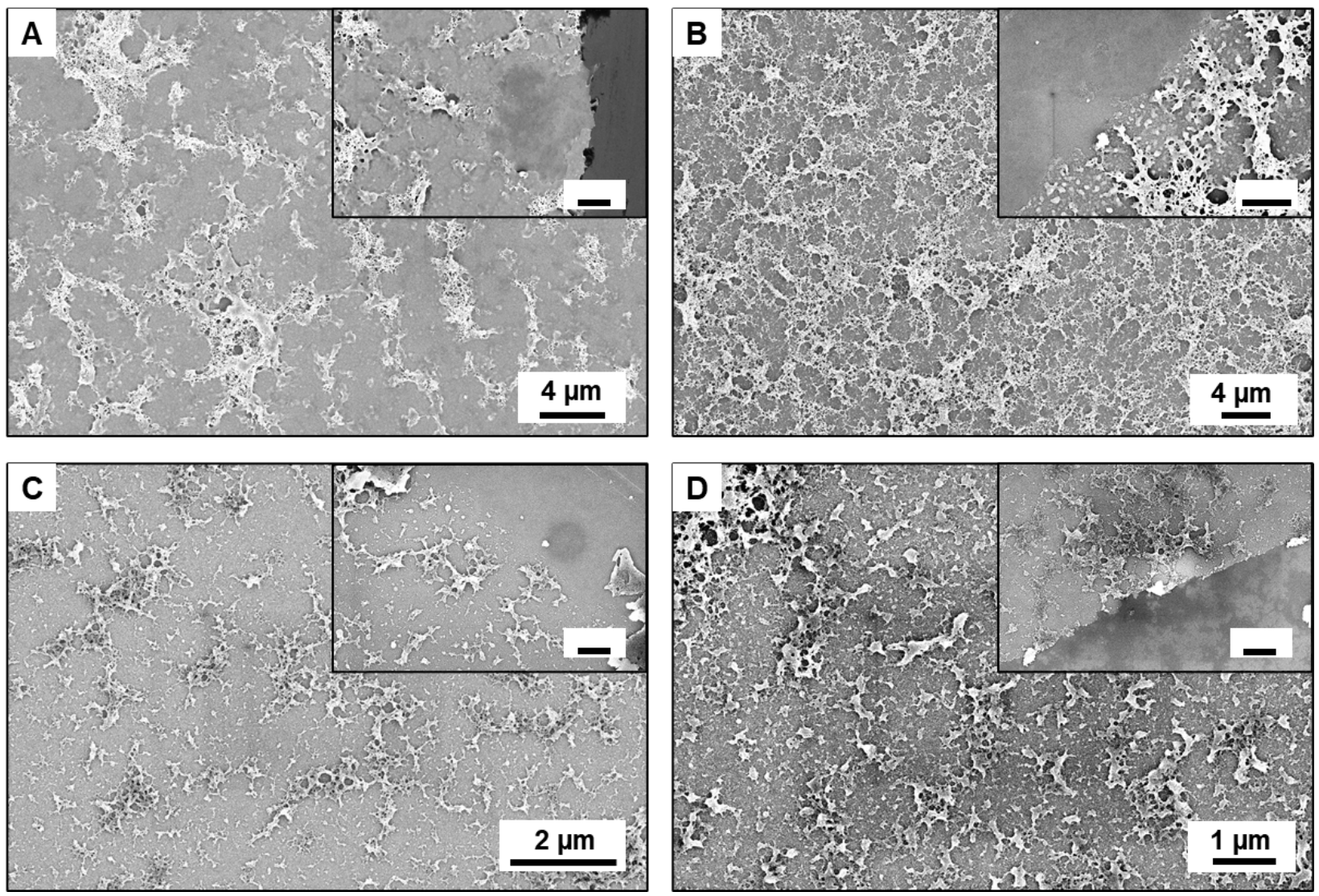

Figure S8. SEM images (top-down) of films fabricated by simultaneously spraying solutions of $\mathbf{P 1}$ and PEI at (A) 1:7, (B) 1:3, (C) 1.9:1, and (D) 3:1 ratios (P1/PEI) of spraying rates onto planar silicon substrates. For all experiments, the combined polymer spraying rate was kept constant at $7.2 \pm 1.2$ $\mathrm{mmol} / \mathrm{s}$. Inset scale bar $=$ (A) $2 \mu \mathrm{m}$, (B) $1 \mu \mathrm{m}$, (C) $400 \mathrm{~nm}$, and (D) $400 \mathrm{~nm}$. 

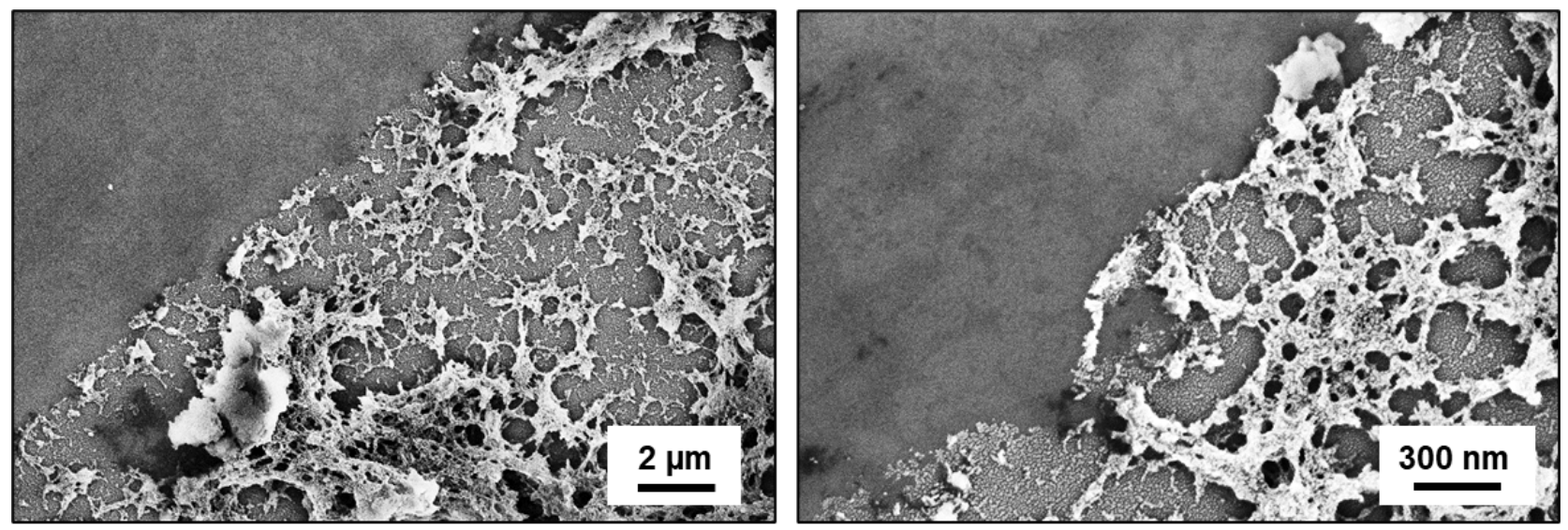

Figure S9. Low- and high-magnification SEM images of films fabricated by simultaneously spraying P1 and PEI at 1:1 ratio of spraying rates on a silicon substrate for 145 seconds. Images show the boundaries between intact films and areas where the films were scratched away. See Materials and Methods section for additional details of film erosion experiments and the acquisition of images by SEM. 

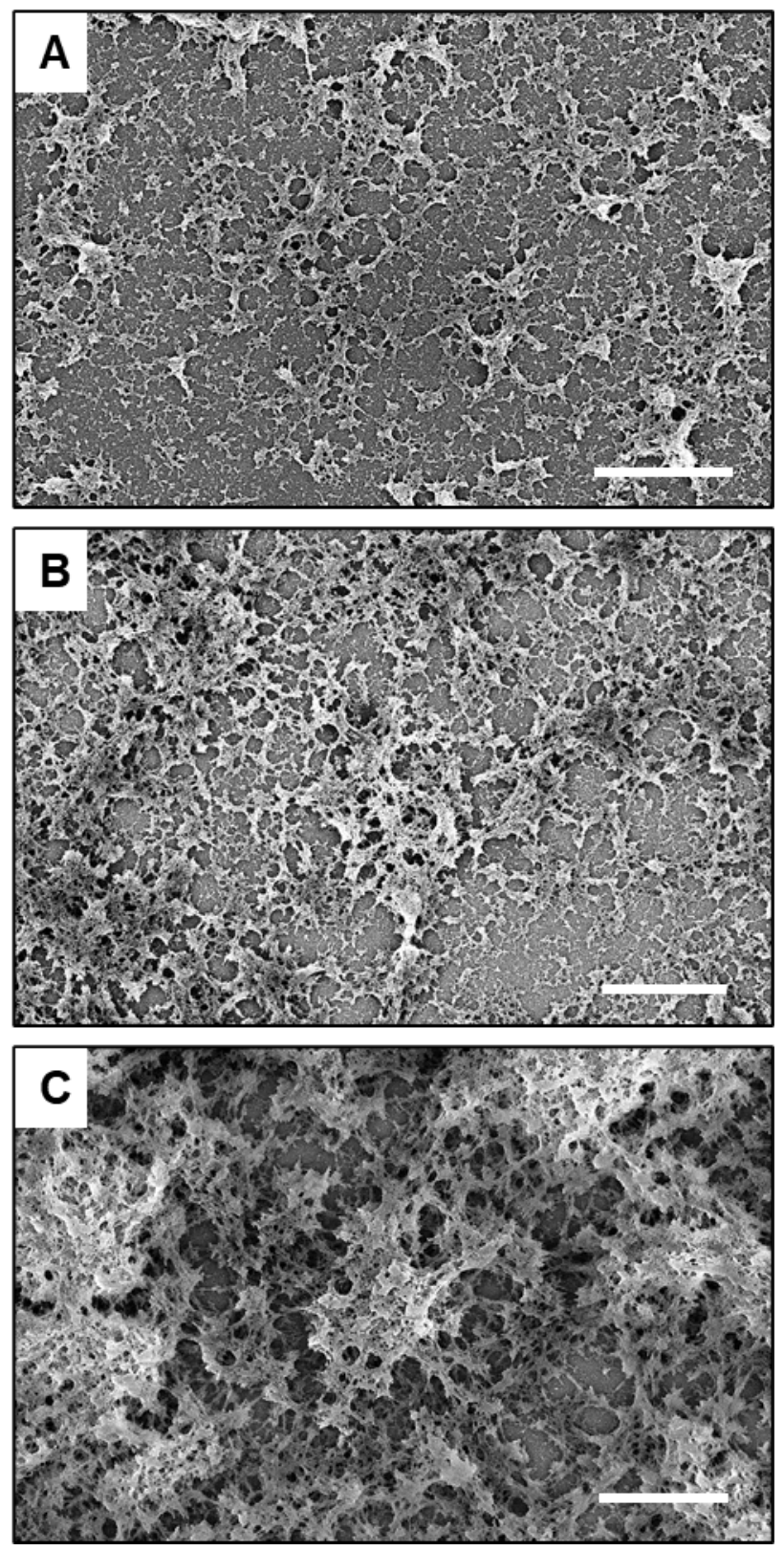

Figure S10. SEM images (top-down) of films fabricated by simultaneously spraying solutions of $\mathbf{P} 1$ and PEI at 1:1 ratio (P1/PEI) of spraying rates after (A) $6 \mathrm{~s}$, (B) $38 \mathrm{~s}$, and (C) $145 \mathrm{~s}$ onto planar silicon substrates. Panel $\mathrm{C}$ is reproduced from the main figures in the paper (Figure 7D). Scale bar $=2 \mu \mathrm{m}$. 


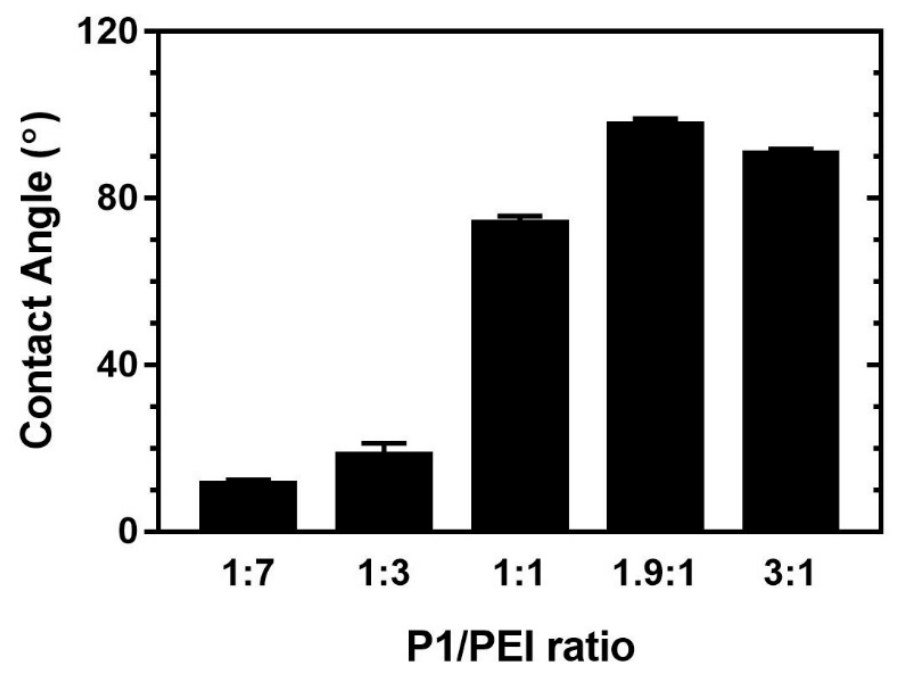

Figure S11. Plot showing contact angle of water droplets $(5 \mu \mathrm{L})$ on films fabricated by simultaneously spraying solutions of $\mathbf{P 1}$ and PEI at different ratios of P1/PEI spraying rates onto planar silicon substrates.
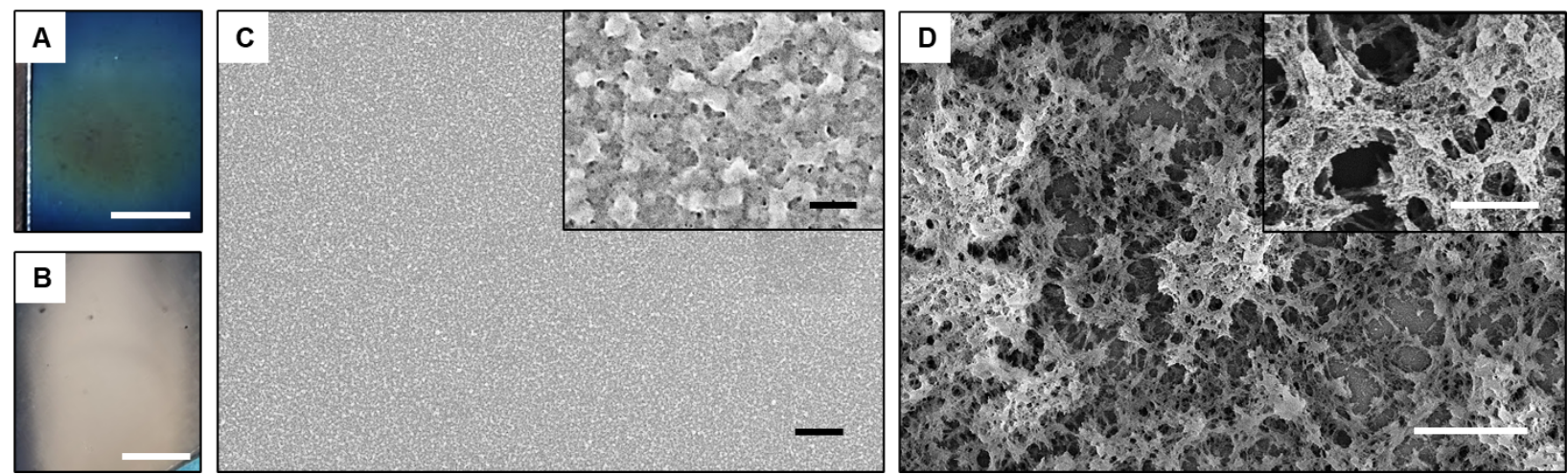

Figure S12. Digital photos (scale bar $=50 \mathrm{~mm}$ ) of PVDMA/PEI (A) and P1/PEI (B) films fabricated by simultaneous spraying onto planar silicon substrates (after 145 seconds of cumulative spraying time). The spraying rate ratio was fixed at 1:1 for both PVDMA/PEI and P1/PEI films. Low- and high-magnification SEM images (top-down) of the PVDMA/PEI (C) and P1/PEI (D) films shown in (A) and (B), respectively. Panel D is reproduced from the main figures in the paper (Figure 7D). Scale bar in $(C)=4$ $\mu \mathrm{m}$ and $(\mathrm{D})=2 \mu \mathrm{m}$, inset scale bars are $400 \mathrm{~nm}$ in both. 


\section{Supporting Videos}

Video S1. Video showing colored aqueous droplets jumping off an $n$-decylamine-treated 35 bilayer P1/PEI film fabricated by sequential spray-based procedures.

Video S2. Video showing a sample of tomato ketchup sliding around a SLIPS-coated glass container. The glass container was spray-coated with 35 P1/PEI bilayers; the multilayers were then reacted with $n$-decylamine and infused with silicone oil to fabricate SLIPS coatings. This video is a companion to the still images shown in Figure 6E-G.

Video S3. Video showing that P1/PEI SLIPS-coated PE tubing segments $(\sim 8 \mathrm{~cm}$ long) are not fouled by blood whereas bare, uncoated PE tubing segments are completely covered in blood. This video is a companion to the still images shown in Figure 6K-N.

Video S4. Video showing the sliding of various chemically complex media on a glass slide $(\sim 2$ $\mathrm{cm}$ long, tilted to $20^{\circ}$ ) coated with silicone oil-infused simultaneously-sprayed P1/PEI films. This video is a companion to the still images shown in Figure 9A.

Video S5. Video showing a droplet of aqueous TMR $(20 \mu \mathrm{L})$ on a sample of SLIPS-coated and uncoated flexible polyester films bent and held end-to-end. This video is a companion to the still images shown in Figure 9B-E.

Video S6. Video showing the sliding of aqueous droplets (colored red and blue to aid visual observation; $15 \mu \mathrm{L}$ each) on SLIPS-coated filter paper. SLIPS coated filter paper was permanently creased prior to placing the aqueous droplets, and the aqueous droplets were observed to contact and slide on the crease. This video is a companion to the still images shown in Figure 9I-K. 